The antiproliferative effect of pasireotide $L A R$ alone and in combination with everolimus in patients with medullary thyroid cancer: a single-center, open-label, phase II, proof-of-concept study Antongiulio Faggiano, Roberta Modica, Rosa Severino, Luigi Camera, Rosa Fonti, et al.

\section{Endocrine}

International Journal of Basic and Clinical Endocrinology

ISSN 1355-008X

Volume 62

Number 1

Endocrine (2018) 62:46-56

DOI 10.1007/s12020-018-1583-7
Volume 62 • Number 1

Octoher 2018

\section{Endocrine}

International Journal of Basic and Clinical Endocrinology

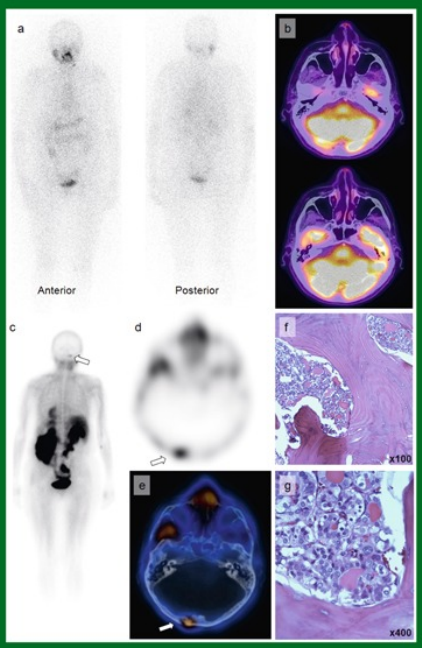

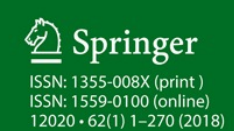

Editor in Chief:

Andrea Giustina 
Your article is protected by copyright and all rights are held exclusively by Springer Science+Business Media, LLC, part of Springer Nature. This e-offprint is for personal use only and shall not be self-archived in electronic repositories. If you wish to selfarchive your article, please use the accepted manuscript version for posting on your own website. You may further deposit the accepted manuscript version in any repository, provided it is only made publicly available 12 months after official publication or later and provided acknowledgement is given to the original source of publication and a link is inserted to the published article on Springer's website. The link must be accompanied by the following text: "The final publication is available at link.springer.com". 


\title{
The antiproliferative effect of pasireotide LAR alone and in combination with everolimus in patients with medullary thyroid cancer: a single-center, open-label, phase II, proof-of-concept study
}

\author{
Antongiulio Faggiano $\mathbb{D}^{1} \cdot$ Roberta Modica $^{1} \cdot$ Rosa Severino $^{2} \cdot$ Luigi Camera $^{2} \cdot$ Rosa Fonti $^{3} \cdot$ Michela Del Prete $^{4,5}$. \\ Maria Grazia Chiofalo ${ }^{6} \cdot$ Massimo Aria $^{7} \cdot$ Piero Ferolla $^{8} \cdot$ Giovanni Vitale $^{9,10} \cdot$ Luciano Pezzullo $^{6} \cdot$ Annamaria Colao $^{1}$
}

Received: 15 December 2017 / Accepted: 14 March 2018 / Published online: 23 March 2018

(c) Springer Science+Business Media, LLC, part of Springer Nature 2018

\begin{abstract}
Purpose Medullary thyroid cancer (MTC) is a neuroendocrine tumour of the thyroid C cells. Pasireotide, a multi-receptor targeted somatostatin analogue, and everolimus, an inhibitor of mTOR, showed antitumour properties in neuroendocrine tumours. Aim of this study was to evaluate pasireotide alone and in combination with everolimus in patients with MTC. Methods Patients with progressive metastatic or persistent postoperative MTC received pasireotide LAR $60 \mathrm{mg} / \mathrm{m}$ for at least 6 months. Patients exhibiting progressive disease received everolimus $10 \mathrm{mg} / \mathrm{d}$ as combination therapy. Primary endpoint was progression free survival (PFS). Secondary endpoints included, overall survival, objective response rates, change in circulating markers, safety. Study registration no. NCT01625520.

Results Nineteen consecutive patients were enrolled. Median follow-up was 31 months. Median PFS with pasireotide was 36 months (95\% CI: 19.5-52.5). Nine patients (47\%) had tumour progression: seven of them started everolimus in combination with pasireotide, achieving a median PFS of 9.0 months (95\% CI: 0-21.83). Five of them (71\%) had further tumour progression, one objective response (14.3\%), one stopped treatment because of pulmonary embolism. Pasireotide alone and with everolimus was safe and required withdrawal only in one case. Diarrhoea and hyperglycaemia were the most frequent adverse events with pasireotide (grade 3 in 5.3\% each). Hyperglycaemia was the most frequent grade 3 toxicity with the combination therapy $(28.6 \%)$.

Conclusions Pasireotide therapy shows antiproliferative effects in persistent postoperative MTC suggesting further investigation on larger series of patients. In progressive MTC lesions, the combination pasireotide plus everolimus may be of benefit. Both schemes were safe and well tolerated.
\end{abstract}

Keywords Medullary thyroid cancer $\cdot$ Pasireotide $\cdot$ Everolimus $\cdot$ Neuroendocrine Tumours

Antongiulio Faggiano

afaggian@unina.it

1 Department of Clinical Medicine and Surgery, Federico II University, Naples, Italy

2 Department of Advanced Biomedical Sciences, Radiology, Section of Diagnostic Imaging, University "Federico II", Naples, Italy

3 Institute of Biostructures and Bioimages - National Research Council, Naples, Italy

4 Department of Radiology and Nuclear Medicine, and Cancer Research Center, Université Laval, Quebec City, Canada

5 Department of Medical Imaging, and Oncology Branch of Research Center, CHU de Québec, Université Laval, 11 côte du Palais, Quebec City (QC) G1R 2J6, Canada

6 Thyroid and Parathyroid Surgery Unit, Istituto Nazionale per lo studio e la cura dei tumori "Fondazione G. Pascale" - IRCCS, Naples, Italy

7 Department of Economics and Statistics, University Federico II, Naples, Italy

8 Department of Medical Oncology, Multidisciplinary NET Group, Umbria Regional Cancer Network and University of Perugia, Perugia, Italy

9 Department of Clinical Sciences and Community Health (DISCCO), University of Milan, Milan, Italy

10 Laboratory of Endocrine and Metabolic Research, Istituto Auxologico Italiano IRCCS, Milan, Italy 


\section{Introduction}

Medullary thyroid cancer (MTC) is a well-differentiated neuroendocrine tumour (NET) of the thyroid parafollicular cells, accounting for 1-2\% of all thyroid cancers. Total thyroidectomy and adequate neck dissection are effective in patients with localized disease. However, many patients with MTC show persistence of disease after surgery [1].

An effective therapy is nowadays available for MTC persistent after surgery. Vandetanib and cabozantinib are molecular targeted agents approved for treatment of MTC. However their use should be weighted by considering tumour progression and risk/benefit ratio for the patient. These drugs should not be considered for patients with no tumour lesions at imaging, as well as in those with asymptomatic not progressive small tumour burden [2].

Somatostatin analogues and mTOR inhibitors have been also taken in consideration in this setting. Octreotide and lanreotide mainly target the somatostatin receptor subtype $(\mathrm{sst})_{2}$, resulting in citotastic effects in well differentiated low proliferating NET. However no significant antiproliferative activity has been reported in MTC [3-5]. Pasireotide (SOM230), a novel somatostatin analogue with highbinding affinity for $\mathrm{sst}_{1}, \mathrm{sst}_{2}, \mathrm{sst}_{3}$ and $\mathrm{sst}_{5}$, could be of benefit in MTC, which are characterized by high expression of sst other than sst $_{2}$ [6]. Pasireotide has been evaluated in well-differentiated grade 1-2 gastroenteropancreatic and lung NET, showing antisecretory and potential antitumour properties [7, 8], while no data are available in MTC, with the exception of a single patient with metastatic MTC and ectopic ACTH syndrome well controlled by pasireotide [9].

Everolimus is an inhibitor of mTOR, a central regulator of growth/proliferation, cellular metabolism and angiogenesis, which is involved in NET development [10]. This compound has shown antitumour benefit in patients with gastroenteropancreatic and lung NET alone and in combination with octreotide LAR [11, 12]. In patients with progressive MTC, treatment with everolimus was first reported to exert antiproliferative activity in case reports [13,14], then in $71 \%$ of cases from a subgroup analysis of a phase 2 trial [15].

The aim of this study was to evaluate the efficacy of pasireotide in patients with progressive metastatic or postoperative persistent MTC. The efficacy of pasireotide in combination with everolimus in patients who progressed under pasireotide alone was also evaluated.

\section{Materials and methods}

\section{Study design and patient eligibility}

The study was designed as a prospective, open-label, phase 2, proof-of-concept study and performed in the
NET Center of Naples, a multidisciplinary Center for the diagnosis and therapy of NET. The study enrolled patients with histopathologically confirmed progressive metastatic or postoperative persistent MTC. Patients were enrolled in accordance with an approved protocol, international standards of good clinical practice, institutional review board approval, and institutional safety monitoring, and written informed consent document was provided by patients or their legal representatives. The study was registered at ClinicalTrials.gov (\#NCT01625520). In October 2011 the study protocol received institutional review board approval and in February 2012 the trial commenced enrollment. Recruitment was completed in July 2014.

To be eligible for the study patients were required to be adult aged at least 18 years with histologically proven MTC, metastatic or loco-regional stage, not susceptible to be cured by surgery or relapsing / persistent after surgery, and at least one lesion that could be accurately measured in at least one dimension as $1.0 \mathrm{~cm}$ or greater by spiral computed tomography scan. Alternatively, patients without clear radiologically measurable lesions but with escalating serum calcitonin levels, assessed once a month for at least three months before study entry, according to RECIST definitions (elevation of the marker for at least 25 $\%$ ) could be eligible [16]. Performance status ECOG $\leq 2$, life expectancy $\geq 6$ months, adequate organ function were also required. In women of childbearing potential a negative serum pregnancy test within 14 days of study entry was required. Patients with a known history of impaired fasting glucose or diabetes mellitus could be included, with close blood glucose and antidiabetic treatment monitoring throughout the trial and adjustments as necessary. Individuals were excluded from participation if they had signs of recurrence of prior or concomitant malignancies (within the last 3 years or requiring active treatment) other than MTC (with the exception of previous basal cell skin cancer, previous cervical carcinoma in situ); unstable systemic diseases, any active or uncontrolled infection/disorders (except for Hep B and Hep C positive patients), liver cirrhosis, chronic active or persistent hepatitis, severely impaired lung function, active bleeding diathes, fatal or life-threatening autoimmune and ischemic disorders, psychiatric condition or laboratory abnormality that may increase the risk associated with study participation or study drug administration, or may interfere with the interpretation of study results, and in the judgement of the investigator would make the patient inappropriate for entry into this study; gallstone disease; congestive heart failure (NYHA Class III or IV), unstable angina, sustained ventricular tachycardia, ventricular fibrillation, clinically significant bradycardia, advanced heart block or a history of acute myocardial infarction 
within the six months preceding enrollment; QTcF at screening $>450 \mathrm{msec}$, history of syncope or family history of idiopathic sudden death, sustained or clinically significant cardiac arrhythmias, risk factors for torsades de pointes; pregnant or breast-feeding patients; known hypersensitivity to somatostatin analogues; prior therapy with mTOR inhibitors; participation in a clinical trial to test an investigational drug within 4 weeks prior to visit 1 . Previous treatments with chemotherapy, radiotherapy, locoregional therapy (eg, liver embolization), octreotide, lanreotide or interferon were permitted providing that toxicity has resolved to < grade 1 at study entry and that last treatment was at least 4 weeks prior to baseline assessment.

\section{Procedures}

Pasireotide LAR $60 \mathrm{mg}$ was administered intramuscularly every $28 \pm 2$ days. Study drug pasireotide LAR i.m. depot injections was supplied by Novartis as a powder in vials containing $20 \mathrm{mg}$ and $40 \mathrm{mg}$ labelled as SOM230 LAR (Novartis Farma S.p.A.), with ampules containing $2 \mathrm{~mL}$ of vehicle (for reconstitution). Oral everolimus (RAD001, Novartis Farma S.p.A.) was administered at the dose of $10 \mathrm{mg}$ per os a day. Study drug everolimus was supplied in open-label packaging by Novartis as tablets of $5 \mathrm{mg}$ strength, blister-packed under aluminum foil in units of 10 tablets and dosed on a daily basis.

All patients started with the same treatment, as a monotherapy with pasireotide LAR i.m. After 3 months of treatment efficacy was evaluated. Patients benefiting from the treatment continued with the monotherapy (stable disease or better according to RECIST 1.1) until progression, start of new cancer therapy, withdrawal of informed consent, occurrence of unacceptable toxicity [16]. Patients progressing were switched to the combination therapy with pasireotide LAR i.m. and oral everolimus. Patients with obvious progressions seen before the 3 months evaluation were switched also. Evaluation of response to the treatment with the combination was after 3 months. Patients were treated for at least 6 months. Patients who profited from the treatment (stable disease or better according to RECIST 1.1) were offered to continue on the study drug during a follow up phase, either in combination or in monotherapy. Followup continued until the patient had documented disease progression according to RECIST 1.1 criteria or started another cancer therapy [16]. For patients who were unable to tolerate the protocol-specified dosing schedule, dose adjustments were permitted in order to keep the patient in the study. Dosage reductions were required in response to preset adverse event parameters. Patients whose treatment was interrupted or permanently discontinued due to an adverse event or abnormal laboratory value were followed at least weekly until resolution or stabilization of the event. Patients discontinued the study if a dose delay of $>1$ month for pasireotide and $\geq 21$ days for everolimus occurred. All patients were followed for adverse events and serious adverse events for 56 days following the last dose of pasireotide LAR and for 28 days following the last dose of everolimus.

Radiological tumour response was assessed by contrastenhanced CT according to RECIST 1.1 criteria. Tumour response was assessed every three months for the first 6 months of pasireotide monotherapy, as well as for the first six months of pasireotide plus everolimus combination therapy. Serum calcitonin and CEA measurements were performed every three months. Commercially available kits were used to assay calcitonin and CEA serum concentrations. Safety was assessed according to the Common Terminology Criteria for Adverse Events (CTCAE) version 4.0. Clinical symptoms and biochemical parameters were evaluated monthly.

\section{Outcomes}

The primary endpoint was the progression free survival (PFS) under treatment with pasireotide LAR, according to RECIST 1.1 [16]. PFS was defined as the time from first study drug administration to objective tumour progression. If a patient has not had an event, PFS was censored at the date of last adequate tumour assessment. Additionally, subjects who started a new anti-cancer therapy prior to documented progression of disease were censored at the date of the last tumour assessment, prior to the start of the new therapy. Secondary endpoints included PFS with pasireotide LAR plus everolimus, objective tumour response rate, overall survival (OS), biochemical response by considering changes from baseline in serum calcitonin and CEA concentrations, and safety. Safety was evaluated using assessment of adverse events and laboratory data. The assessment of safety was based on the frequency of adverse events and on the number of laboratory values that are new or worsening based on CTCAE v4.0. Other safety data (e.g., vital signs) were considered appropriately.

\section{Statistical methods}

Primary efficacy and safety analyses were conducted at end of study for all patients. For all efficacy analyses, the ITT population was used. For all safety analyses, the safety population was used. The ITT population consisted of all patients that received at least one dose of study drug and had at least one post-baseline assessment of the primary efficacy variable. PFS was analysed explored graphically by using the Kaplan-Meier survival estimates. No interim analysis was planned. The Safety Population consisted of all 
patients that received at least one dose of study drug and was analysed according to treatment received. Of note, the statement that a patient had no adverse events also constituted a safety assessment.

\section{Results}

\section{Patient characteristics}

As of 31 January 2017, the date of data cutoff for the primary analysis, 23 patients had been screened and 19 eligible patients had been enrolled and treated with pasireotide. Four out of the 23 screened patients did not meet inclusion criteria $(n=3)$ or decline to participate the study $(n=1)$. All the 19 enrolled patients completed at least six months of treatment with pasireotide. Median follow-up was 31 months (range, 6-59). Median follow-up with pasireotide alone was 26 months (range, 3-59). Patient characteristics at baseline are shown in Tables 1 and 2. At the time of the primary analysis, treatment was still ongoing in eight patients, seven of them with pasireotide alone and one of them with pasireotide and everolimus in combination.

Total thyroidectomy plus lymphadenectomy was performed in all patients and nodal involvement was positive in all of them but one. At enrollment, 15 patients had measurable tumour lesions according to RECIST 1.1 (79\%). Four patients were not found to harbour measurable lesions but encountered the RECIST 1.1 criteria for tumour marker progression $(21 \%)$.

${ }^{111}$ In-Octreotide single photon emission tomography $\left({ }^{111}\right.$ In-Octreotide SPECT) was performed in all patients by using using the commercially available kit OctreoScan (Covidien Italia S.p.A., Segrate, Milan, Italy). Tumour uptake was scant, being negative in 9 of 19 and weakly positive in the remaining 10 (Krenning score 1-2 in 8 cases and score 3 in 2 cases).

\section{Efficacy assessment}

\section{Monotherapy with pasireotide}

Median PFS with pasireotide alone was 36 months $(95 \%$ CI: 19.5-52.5) (Fig. 1). Nine patients out of the 19 had tumour progression (47\%): seven of them started everolimus in combination with pasireotide. Two patients decided to interrupt the trial and did not start everolimus. Among the ten patients who did not experience progression under pasireotide alone, one discontinued the treatment due to the occurrence of a second cancer (colon cancer) at 22month follow-up. Two other patients discontinued the treatment at 6 and 12-month follow-up. None of the patients treated with pasireotide in monotherapy experienced an objective tumour response (Fig. 2).

The median PFS was significantly lower in patients with time to tumour progression before the study $\leq 1$ year than in those with time to progression $>1$ year $(\mathrm{p}<0.05)$, as well as in patients with doubling time of serum calcitonin and CEA levels $\leq 1$ year before the study than in those with doubling time $>1$ year $(p<0.001)$. At the multivariate analysis, the doubling time of CEA was the best predictor of progression (ExpB: 0.38, $p=0.003$ ).

A significant decrease of serum calcitonin concentrations was observed in five patients $(26 \%)$, while progression in six $(32 \%)$. In eight other cases $(42 \%)$ calcitonin concentrations did not varied significantly (Fig. 3). A significant decrease of serum CEA concentrations was observed in two patients (10\%), progression in $11(58 \%)$, stability in the remaining six cases (32\%) (Fig. 3). In $10 \%$ of cases a biochemical response was observed for both markers. Serum calcitonin and CEA variations were not significantly associated to radiological changes $(p=0.43 ; p$ $=0.14$, respectively).

Median PFS was similar between patients with positive and negative ${ }^{111}$ In-Octreotide SPECT tumour uptake ( $p=$ $0.19)$.

\section{Combination therapy pasireotide plus everolimus}

In the seven patients treated with pasireotide plus everolimus, the median PFS was 9.0 months (95\% CI: 0-21.83): five of them had further tumour progression, while one patient discontinued the treatment for the occurrence of pulmonary embolism. An objective tumour response was observed in one patient, 6 months after starting the combination therapy.

Two patients had a significant decrease of both serum calcitonin and CEA concentrations, while four other patients had significant progression of both markers. One patient showed both calcitonin and CEA stability. Serum calcitonin and CEA variations were not significantly associated to radiological changes $(p=0.81)$.

\section{Overall survival (OS)}

Among the 19 patients enrolled in the study, the median overall survival was not reached, while survival rate at 6 and 36 months was 95 and $84 \%$. Five patients (26\%) died of disease after completing the study. Among the seven patients treated with pasireotide plus everolimus, the median OS was 41 months. At the time of primary analysis, four patients were alive, and three have died of disease after completing the study. 


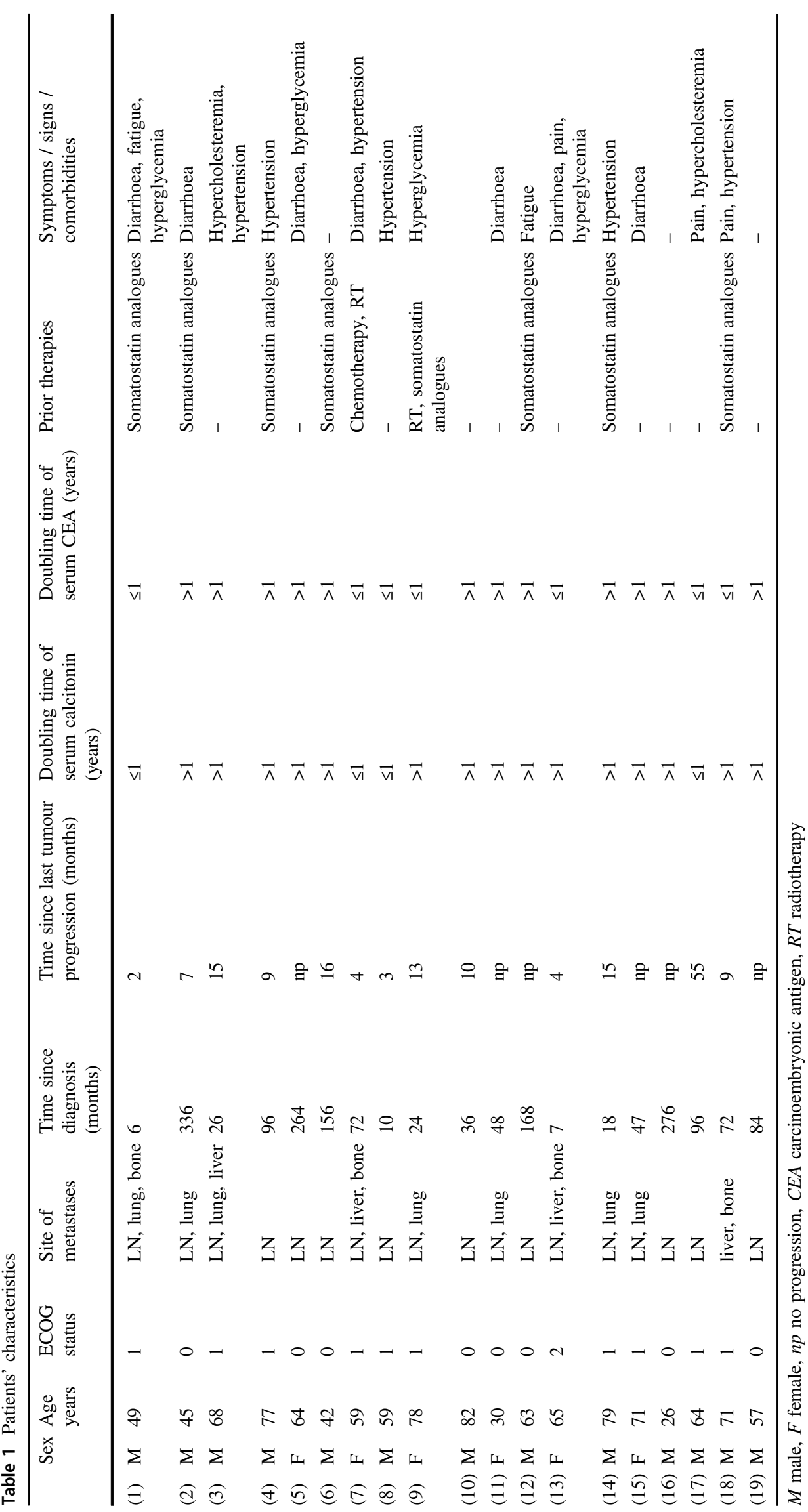


Table 2 Patients' characteristics: cumulative data

\begin{tabular}{|c|c|}
\hline Characteristics & Patients $(n=19)$ \\
\hline \multicolumn{2}{|l|}{ Age at enrollment (yr) } \\
\hline Mean & 60.5 \\
\hline Median (range) & $64(26-82)$ \\
\hline \multicolumn{2}{|l|}{ Sex } \\
\hline Male & 13 \\
\hline Female & 6 \\
\hline \multicolumn{2}{|l|}{ RET status } \\
\hline Positive & 0 \\
\hline Negative & 19 \\
\hline \multicolumn{2}{|l|}{ ECOG performance status } \\
\hline 0 & 8 \\
\hline 1 & 10 \\
\hline 2 & 1 \\
\hline \multicolumn{2}{|l|}{ Site of metastases } \\
\hline Lymph node & 18 \\
\hline Liver & 4 \\
\hline Bone & 4 \\
\hline Lung & 7 \\
\hline \multicolumn{2}{|l|}{ Time since diagnosis (months) } \\
\hline Mean & 97 \\
\hline Median (range) & $72(6-336)$ \\
\hline \multicolumn{2}{|l|}{ Time since last tumour progression } \\
\hline$\leq 1$ year & 8 \\
\hline$>1$ year* & 11 \\
\hline \multicolumn{2}{|c|}{ Doubling time of serum calcitonin concentrations } \\
\hline$\leq 1$ year & 4 \\
\hline$>1$ year & 15 \\
\hline \multicolumn{2}{|c|}{ Doubling time of serum CEA concentrations } \\
\hline$\leq 1$ year & 7 \\
\hline$>1$ year & 12 \\
\hline \multicolumn{2}{|l|}{ Prior therapies } \\
\hline Radiotherapy & 2 \\
\hline Chemotherapy & 1 \\
\hline Biotherapy & 8 \\
\hline \multicolumn{2}{|c|}{ Symptom/Signs/Comorbidities at enrollment } \\
\hline Diarrhoea grade $1 / 2$ & $2 / 5$ \\
\hline Pain grade $1 / 2$ & $2 / 1$ \\
\hline Fatigue grade $1 / 2$ & $1 / 1$ \\
\hline Hyperglycemia grade 1 & 4 \\
\hline Hypercholesteremia grade 1 & 2 \\
\hline Arterial hypertension grade $1 / 2$ & $5 / 1$ \\
\hline
\end{tabular}

*Six patients had no tumour progression after the last surgery

$C E A$ carcinoembryonic antigen

\section{Clinical response and safety assessment}

\section{Monotherapy with pasireotide}

The most CTCAE grades 3 toxicities were diarrhoea (5.3\%) and hyperglycaemia (5.3\%) (Table 3 ). Both were managed without need to stop or decrease pasireotide in any patient. In one patient pasireotide was stopped because of the development of a non-treatment related secondary malignancy (i.e., colon cancer). No CTCAE grades 4-5 toxicities occurred.

\section{Combination therapy pasireotide plus everolimus}

The CTCAE grades 3 toxicities were hyperglycaemia (28.6\%), fatigue (14.3\%) and dyspnoea (14.3\%) (Table 4). The patient with grade 3 dyspnoea had a diagnosis of pulmonary embolism and therefore discontinued the treatment with everolimus. None of the other patients need to stop the treatment. No CTCAE grades 4-5 toxicities occurred.

\section{Discussion}

MTC is a rare NET which frequently persists after surgery. It generally growths slowly metastasizing to neck and mediastinum lymph nodes as well as to bone, liver and more rarely to other organs $[1,2]$. If not cured by surgery, MTC represents a challenge for physicians because there is currently a lack of available therapies with proven survival benefit for MTC. In patients with distant metastases, a 5year survival of $26 \%$ is reported [17]. Two molecular targeted agents, vandetanib and cabozantinib, have recently been shown to impact on PFS in patients with advanced MTC [18, 19]. However, their use should be restricted to patients with significant tumour burden and well-established tumour progression, in order to achieve a positive risk/ benefit ratio [2].

In many patients with postoperative MTC persistence, the disease activity is indicated, at the beginning, by high and progressively increasing circulating levels of calcitonin only without radiologically evident metastases and then, often after many years, it results in a slow metastatic spread. Nowadays, there is no effective therapy to treat and cure postoperative persistent MTC patients before the occurrence of distant metastases. In this setting, to arrest the disease at an early stage when it is characterized by biochemical progression only or even in case of small non-progressive tumour lesions may be effective to prevent the subsequent development of distant metastases. Somatostatin analogues could be an interesting option at this purpose, since they have been demonstrated to be active in NET [20, 21]. The low side effect profile of this category of compounds may be helpful to treat MTC patients who present with asymptomatic disease. Octreotide and lanreotide act preferentially through the sst $_{2}$. In MTC, these compounds have been reported to exert anti-secretive effects on calcitonin but no anti-proliferative effects [3-5]. One possible explanation for this poor response is to find in the peculiar profile of sst 
Fig. 1 Kaplan-Meier estimate of progression-free survival

measured in all patients $(n=19)$ from pasireotide treatment initiation to disease progression or death, whichever occurred first. Vertical lines show censored events. RECIST $=$ Response Evaluation Criteria in Solid Tumours. The median progression-free survival was 36 months (95\% CI: $19.5-52.5$ ). As of 31 January 2017, a total of nine events of disease progression or death had occurred

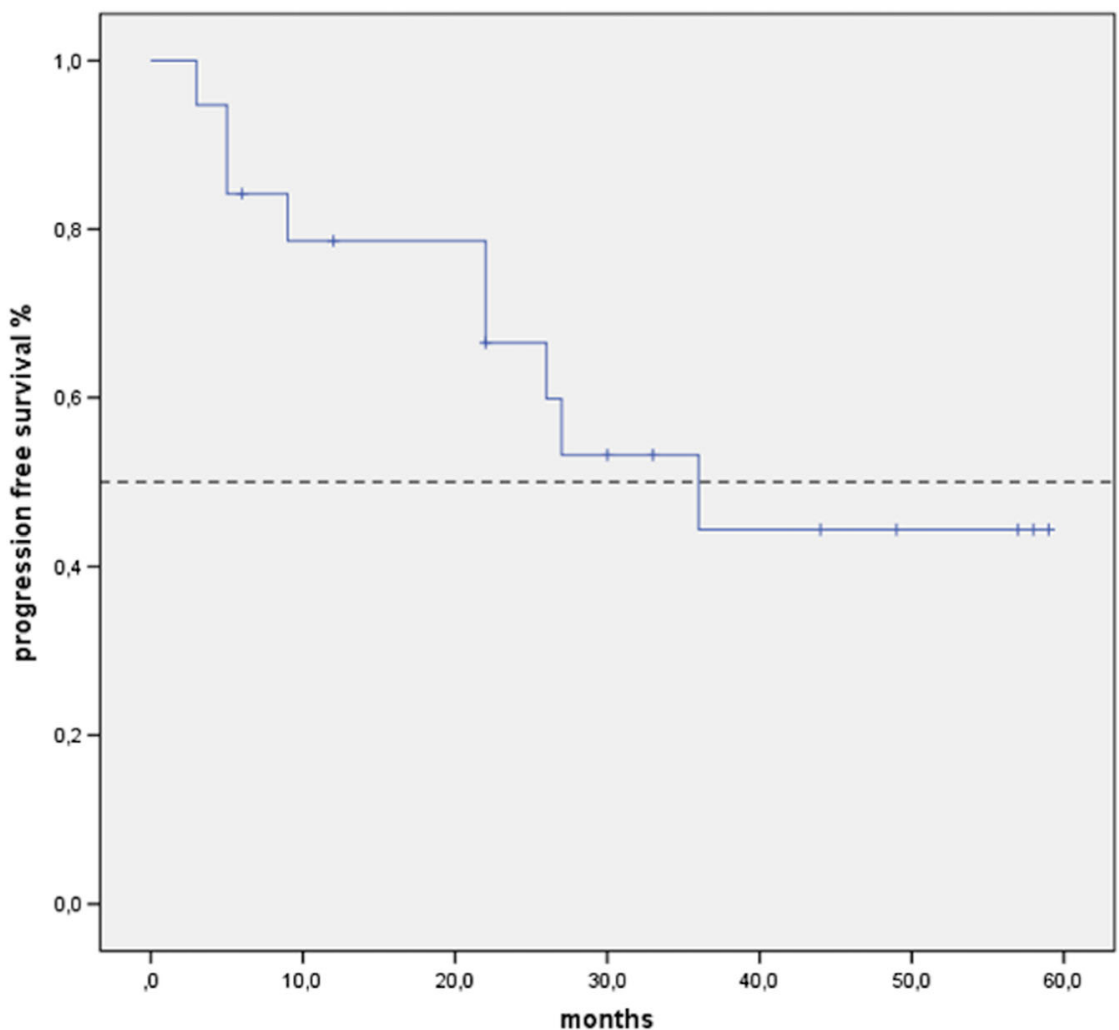

expression in MTC where $\mathrm{sst}_{1}, \mathrm{sst}_{3}, \mathrm{sst}_{5}$ are expressed at least as $\mathbf{s s t}_{2}$ [6]. For this reason, pasireotide, a new somatostatin analogue with high affinity for $\mathrm{sst}_{1}, \mathrm{sst}_{2}, \mathrm{sst}_{3}, \mathrm{sst}_{5}$ can be effective as antiproliferative agent in MTC. No data are available by now about therapy with pasireotide in MTC, except for a clinical case reporting on the effectiveness of this compound in controlling a MTC-related ectopic ACTH syndrome [9]. The current study is the first one investigating the role of pasireotide in patients with MTC. Although no objective response was observed, the median PFS with pasireotide was 36 months. In the phase 3 trial with vandetanib, Wells and coworkers reported a median PFS of 30.5 months in the treatment group and 19.3 in the placebo group [19]. With the due differences regarding study design and sample amplitude, this study is comparable to the current one in terms of study population because both included patients regardless of tumour progression. Therefore the prolongation of PFS to 36 months in patients who received pasireotide should be considered relevant. Pasireotide resulted to be not effective in aggressive tumours, suggesting an antiproliferative role of this agent in those cases with small not progressive lesions or in those with biochemical persistence but not tumour evidence at imaging. On the contrary, pasireotide has not to be used in progressive MTC.
In this study, sst imaging uptake was not predictive of response to pasireotide. This is not surprising if we consider the sst profile of MTC as well as the binding profile of pasireotide. Further studies need to be performed to assess pasireotide activity in vivo in relation to sst expression on MTC tissue samples. These data could be useful to select the best candidate for therapy with pasireotide in MTC. On the other hand, it has to be underlined that therapy with somatostatin analogues has been also used in NET independently by the sst imaging score, because these agents also exert indirect effects not related to binding to sst in tumour cells.

Finally the median OS was not reached in this study. This result could be explained by the fact that more than half patients were not progressive the year before enrollment. It would be interesting, anyway, to further evaluate this point in a dedicated trial.

With regard to the combination therapy with pasireotide and everolimus, promising results were observed consisting in a median PFS of 9.0 months and an objective response rate of $14.3 \%$. These results are comparable with those reported by Schneider et al. in a subgroup analysis of a phase 2 trial performed in advanced thyroid cancer [15]. In this setting, seven MTC patients, all in radiological progression, were treated with everolimus showing stable 

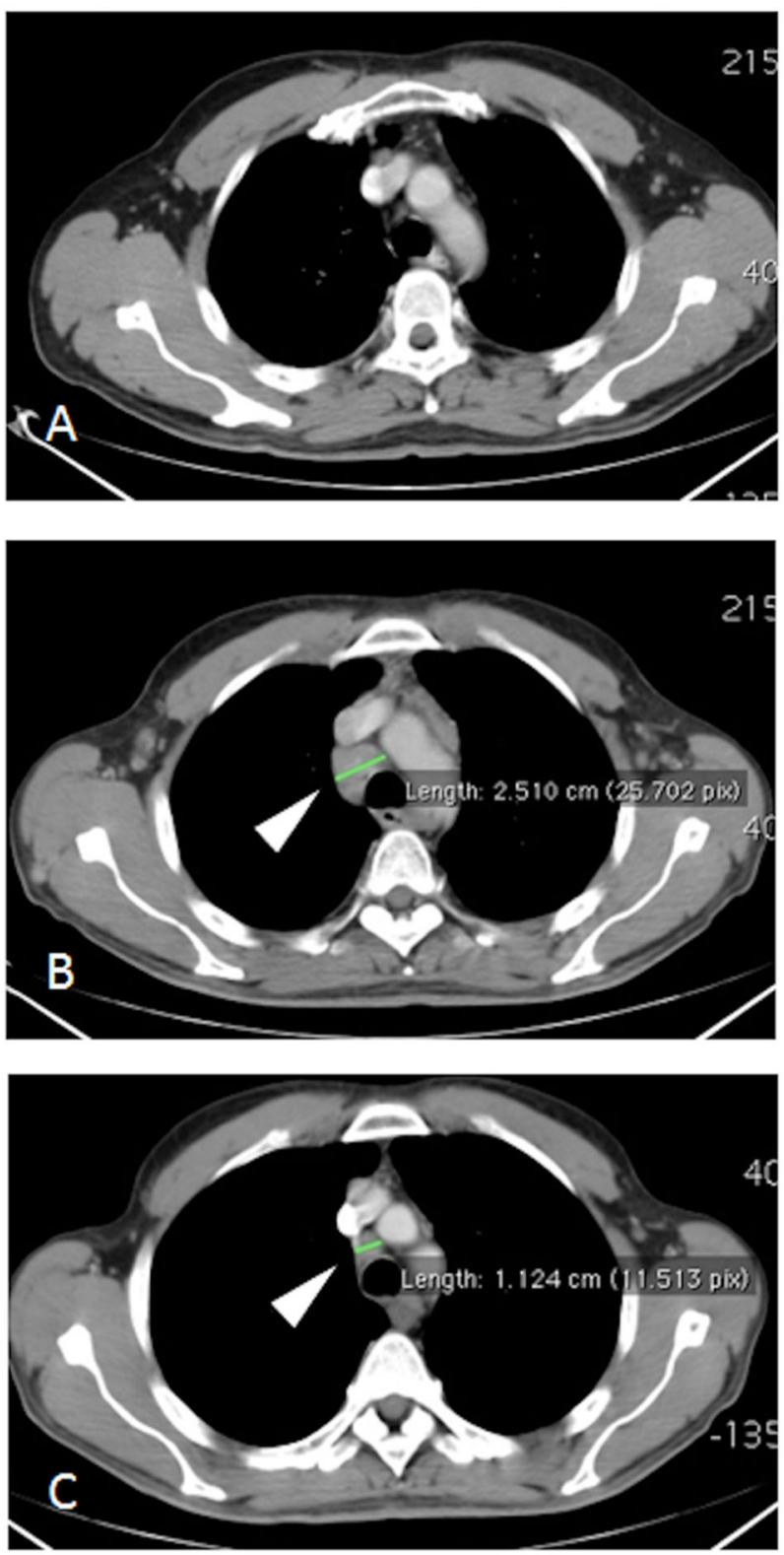

Fig. 2 Contrast-enhanced multi-detector CT in a 56 yrs old male with metastatic MTC. Transverse $5 \mathrm{~mm}$ thick images of mediastinum are shown at the baseline evaluation (a), on pasireotide alone (b) and on pasireotide + Everolimus therapy (c). While no adenopathies are depicted at the baseline evaluation (a), both axillary as well as mediastinal adenopaties can be appreciated in B with the largest crosssectional diameter in the Barety lodge (arrow-head). After 6 month of Pasireotide + Everolimus therapy, a significant $(>50 \%)$ reduction in the largest diameter of the target lesion is depicted indicating a partial response according to RECIST 1.1 criteria

disease in $71 \%$ and a median PFS of about 7.7 months. Study populations were identical for number of patients and tumour progression, while they differed for combination with pasireotide only in the current study and previous treatment with tyrosine kinase inhibitors only in the Schneider study [15]. These differences could explain the slight better results in our study. Previous in vivo and in vitro studies suggested that everolimus with somatostatin analogues have synergistic effects. The first evidence of clinical activity of everolimus on clinical and tumour response was reported in two patients with advanced progressive MTC treated with a combination therapy everolimus plus octreotide [14]. In vitro studies on MTC cell lines supported a significant antitumour activity of everolimus in this setting, not only by inhibiting cell proliferations but also inducing senescence $[14,22]$, while the combination of octreotide/pasireotide with everolimus was more powerful to inhibiting cell proliferation in NET cell lines than either agent alone [23].

A phase 2 trial with pazopanib, a multi-target tirosine kinase inhibitor, showed similar results as the current study in 35 advanced MTC with tumour progression at enrolment [24]. The median PFS was 9.4 months and the objective response rate was $14.3 \%$. However, the median OS was 19.9 months in this study, with 22 of 35 patients died of disease at the time of data lock [24]. In the current study, the overall survival since the time of everolimus start to the time of primary analysis was 41 months. A possible explanation for this difference was that our patients were naïve for targeted therapies which could be used after progression with pasireotide and everolimus. There is a still higher difference if we compare the current study with that by Schneider et al., where the seven MTC patients had a median overall survival of 30 weeks, being for the most pre-treated with tyrosine kinase inhibitors [15].

Together with the anti-tumour efficacy, both pasireotide monotherapy and everolimus were also effective on calcitonin and CEA serum concentrations. Nevertheless, there was no concordance between serum marker and radiological changes, in line with previous studies [15]. This suggests that antitumour and antisecretory activity of pasireotide, as well as everolimus, could be independent and neither calcitonin nor CEA can be reliable predictors of MTC response to these drugs.

As expected, pasireotide was well tolerated. Adverse events were for the most grade 1-2 and did not require drug interruption or discontinuation. Hyperglycaemia was the most common side effect, which was effectively managed by anti-diabetes regimens and drugs. This optimal safety profile fits well with the possible role of pasireotide in asymptomatic not progressive MTC patients. The combination therapy with everolimus was associated to a worsened safety profile. The higher rate of hyperglycaemia as compared to the other everolimus MTC study is clearly due to the synergistic effects of pasireotide and everolimus on glucose homeostasis [15]. However, no grade 4 toxicity was observed, and the safety profile remained better than pazopanib, vandetanib and cabozantinib [18, 19, 24]. 
Fig. 3 Percentage change in serum calcitonin and carcinoembrionic antigen (CEA) variation from baseline over time for all patients $(n=19)$ Upper dotted line represents progression at $50 \%$ and lower dotted line represents the

RECIST boundary for biochemical response at $50 \%$
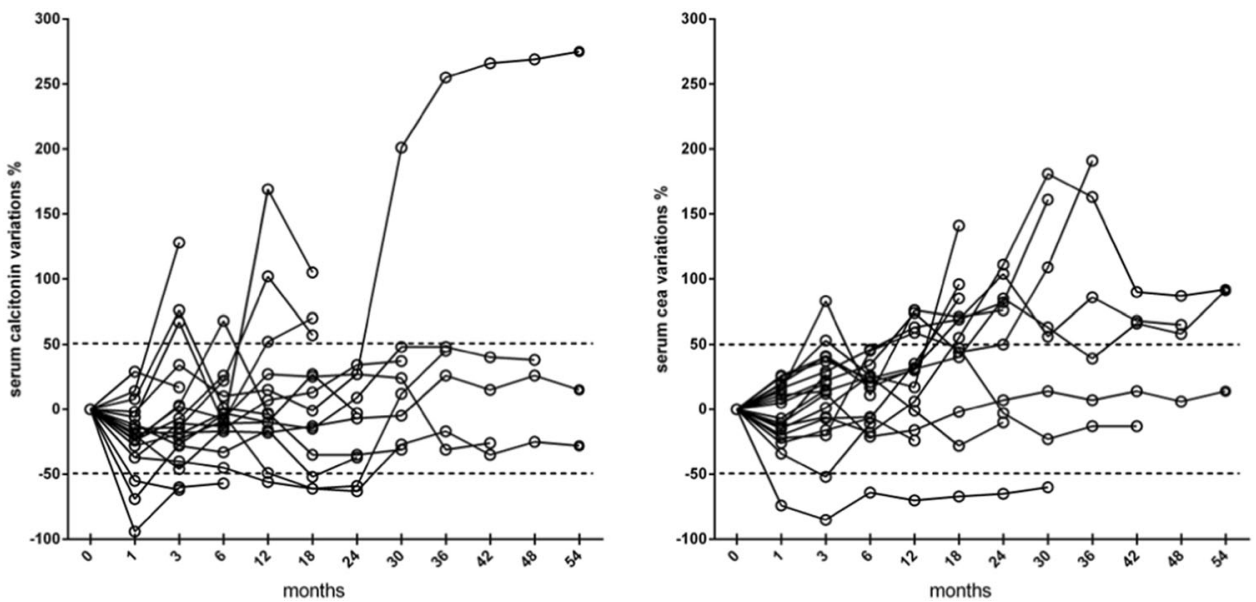

Table 3 Pasireotide adverse events (patients $n=19$ )

Table 4 Pasireotide plus everolimus adverse events (patients, $n=7$ )

\begin{tabular}{|c|c|c|c|c|c|}
\hline Adverse events & Grade 1 & Grade 2 & Grade 3 & Grade 4 & Grade 5 \\
\hline Fatigue & & 1 & & & \\
\hline Depression & 2 & & & & \\
\hline Pain & 2 & 1 & & & \\
\hline Diarrhoea & 9 & 2 & 1 & & \\
\hline Nausea & 2 & & & & \\
\hline Hyperglycemia & 8 & 4 & 1 & & \\
\hline Hypercholesterolemia & 1 & & & & \\
\hline Hypocalcemia & 1 & & & & \\
\hline Rash & 1 & & & & \\
\hline Alanine aminotransferase increased & 1 & & & & \\
\hline Aspartate aminotransferase increased & 1 & & & & \\
\hline Cholelitiasis & 1 & & & & \\
\hline Adrenal insufficiency & & 1 & & & \\
\hline Secondary malignancy & & & & 1 & \\
\hline
\end{tabular}

\begin{tabular}{|c|c|c|c|c|c|}
\hline Adverse events & Grade 1 & Grade 2 & Grade 3 & Grade 4 & Grade 5 \\
\hline Anaemia & 1 & & & & \\
\hline Leukopenia & 1 & & & & \\
\hline Fatigue & 1 & & 1 & & \\
\hline Depression & 1 & & & & \\
\hline Pain & 1 & 1 & & & \\
\hline Diarrhoea & 1 & 2 & & & \\
\hline Nausea & 1 & & & & \\
\hline Hyperglycemia & & 2 & 2 & & \\
\hline Hypercholesterolemia & 1 & & & & \\
\hline Hypertriglyceridemia & 1 & & & & \\
\hline Hypocalcemia & 1 & & & & \\
\hline Mucositis/Stomatitis & 2 & & & & \\
\hline Dysphonia & & 1 & & & \\
\hline Dyspnea & & & 1 & & \\
\hline Alanine aminotransferase increased & 1 & & & & \\
\hline Aspartate aminotransferase increased & 1 & & & & \\
\hline
\end{tabular}




\section{Conclusions}

Pasireotide LAR could represent an effective and safe systemic treatment, in order to delay tumour progression, in patients with postoperative persistent MTC with small not progressive lesions or biochemical progression only. To combine pasireotide with everolimus in patients with progressive MTC could be an intriguing possible approach. The definitive role of pasireotide therapy, as well as the combination therapy pasireotide plus everolimus in MTC, remain to be established in randomized trials on larger series of patients.

Author contributions A.F.: conceived the study design and wrote the article. R.M., G.P., M.D.P., M.G.C., P.F.: collected clinical and biochemical data. L.C., R.S., R.F.: analyzed the radiological tumour response and wrote the part related to imaging. G.V., L.P.: proof-read the manuscript. A.C.: supervised the project

\section{Compliance with ethical standards}

Conflict of interest A. Faggiano and A. Colao Department received grant for research from Novartis. The remaining authors declare that they have no conflict of interest.

Ethical approval All procedures performed in studies involving human participants were in accordance with the ethical standards of the institutional and/or national research committee and with the 1964 Helsinki declaration and its later amendments or comparable ethical standards.

Informed consent Informed consent was obtained from all individual participants included in the study.

\section{References}

1. J. Hadoux, F. Pacini, R.M. Tuttle, M. Schlumberger, Management of advanced medullary thyroid cancer. Lancet Diabetes Endocrinol. 4, 64-71 (2016)

2. S.A. Wells Jr., S.L. Asa, H. Dralle, R. Elisei, D.B. Evans, R.F. Gagel, N. Lee, A. Machens, J.F. Moley, F. Pacini, F. Raue, K. Frank-Raue, B. Robinson, M.S. Rosenthal, M. Santoro, M. Schlumberger, M. Shah, S.G. Waguespack; American Thyroid Association Guidelines Task Force on Medullary Thyroid Carcinoma., Revised American Thyroid Association guidelines for the management of medullary thyroid carcinoma. Thyroid $\mathbf{2 5}$, 567-610 (2015)

3. K. Frank-Raue, F. Raue, R. Ziegler, Therapy of metastatic medullary thyroid gland carcinoma with the somatostatin analog octreotide. Med. Klin. 90, 63-66 (1995)

4. E. Modigliani, R. Cohen, S. Joannidis, C. Siame-Mourot, J.M. Guliana, G. Charpentier, D. Cassuto, M. Bentata Pessayre, A. Tabarin, P. Roger, P. Caron, P.J. Gullasseau, J.D. Lalau, J. Tournlafre, F. Bayard, P. Aulevre, A. James-Deldier, C. Catmettest, Results of long-term continuous subcutaneous octreotide administration in 14 patients with medullary thyroid carcinoma. Clin. Endocrinol. 36, 183-186 (1992)

5. G. Vitale, P. Tagliaferri, M. Caraglia, E. Rampone, A. Ciccarelli, A.R. Bianco, A. Abbruzzese, G. Lupoli, Slow release lanreotide in combination with interferon-alpha2b in the treatment of symptomatic advanced medullary thyroid carcinoma. J. Clin. Endocrinol. Metab. 85, 983-988 (2000)

6. M.C. Zatelli, D. Piccin, F. Tagliati, A. Bottoni, A. Luchin, C. Vignali, A. Margutti, M. Bondanelli, G.C. Pansini, M.R. Pelizzo, M.D. Culler, E.C. Degli Uberti, Selective activation of somatostatin receptor subtypes differentially modulates secretion and viability in human medullary thyroid carcinoma primary cultures: potential clinical perspectives. J. Clin. Endocrinol. Metab. 91, 2218-2224 (2006)

7. M. Cives, P.L. Kunz, B. Morse, D. Coppola, M.J. Schell, T. Campos, P.T. Nguyen, P. Nandoskar, V. Khandelwal, J.R. Strosberg, Phase II clinical trial of pasireotide long-acting repeatable in patients with metastatic neuroendocrine tumors. Endocr. Relat. Cancer 22, 1-9 (2015)

8. L.K. Kvols, K.E. Oberg, T.M. O'Dorisio, P. Mohideen, W.W. de Herder, R. Arnold, K. Hu, Y. Zhang, G. Hughes, L. Anthony, B. Wiedenmann, Pasireotide (SOM230) shows efficacy and tolerability in the treatment of patients with advanced neuroendocrine tumors refractory or resistant to octreotide LAR: results from a phase II study. Endocr. Relat. Cancer 19, 657-666 (2012)

9. F.A. Verburg, M. Anlauf, F.M. Mottaghy, W. Karges, Somatostatin receptor imaging-guided pasireotide therapy in medullary thyroid cancer with ectopic adrenocorticotropin production. Clin. Nucl. Med. 40, e83-e84 (2015)

10. M.C. Zatelli, G. Fanciulli, P. Malandrino, V. Ramundo, A. Faggiano, A. Colao; Group Nike., Predictive factors of response to mTOR inhibitors in neuroendocrine tumours. Endocr. Relat. Cancer 23, R173-R183 (2016)

11. J.C. Yao, N. Fazio, S. Singh, R. Buzzoni, C. Carnaghi, E. Wolin, J. Tomasek, M. Raderer, H. Lahner, M. Voi, L.B. Pacaud, N. Rouyrre, C. Sachs, J.W. Valle, G.D. Fave, E. Van Cutsem, M. Tesselaar, Y. Shimada, D.Y. Oh, J. Strosberg, M.H. Kulke, M.E. Pavel; RAD001 in Advanced Neuroendocrine Tumours, Fourth Trial (RADIANT-4) Study Group., Everolimus for the treatment of advanced, non-functional neuroendocrine tumours of the lung or gastrointestinal tract (RADIANT-4): a randomised, placebocontrolled, phase 3 study. Lancet 387, 968-977 (2016)

12. J.C. Yao, M.H. Shah, T. Ito, C.L. Bohas, E.M. Wolin, E. Van Cutsem, T.J. Hobday, T. Okusaka, J. Capdevila, E.G. de Vries, P. Tomassetti, M.E. Pavel, S. Hoosen, T. Haas, J. Lincy, D. Lebwohl, K. Öberg; RAD001 in Advanced Neuroendocrine Tumors, Third Trial (RADIANT-3) Study Group., Everolimus for advanced pancreatic neuroendocrine tumors. N. Engl. J. Med. 364, 514-523 (2011)

13. M. Druce, T.T. Chung, S. Grozinsky-Glasberg, D.J. Gross, A.B. Grossman, Preliminary report of the use of everolimus in a patient with progressive medullary thyroid carcinoma. Clin. Endocrinol. 77, 154-155 (2012)

14. A. Faggiano, V. Ramundo, A. Dicitore, S. Castiglioni, M.O. Borghi, R. Severino, P. Ferolla, L. Crino, A. Abbruzzese, P. Sperlongano, M. Caraglia, D. Ferone, L. Hofland, A. Colao, G. Vitale, Everolimus is an active agent in medullary thyroid cancer: a clinical and in vitro study. J. Cell. Mol. Med. 16, 1563-1572 (2012)

15. T.C. Schneider, D. de Wit, T.P. Links, N.P. van Erp, J.J. van der Hoeven, H. Gelderblom, T. van Wezel, R. van Eijk, H. Morreau, H.J. Guchelaar, E. Kapiteijn, Beneficial effects of the mTOR inhibitor everolimus in patients with advanced medullary thyroid carcinoma: subgroup results of a phase II trial. Int. J. Endocrinol. 2015, 348124 (2015)

16. E.A. Eisenhauer, P. Therasse, J. Bogaerts, L.H. Schwartz, D. Sargent, R. Ford, J. Dancey, S. Arbuck, S. Gwyther, M. Mooney, L. Rubinstein, L. Shankar, L. Dodd, R. Kaplan, D. Lacombe, J. Verweij, New response evaluation criteria in solid tumours: revised RECIST guideline (version 1.1). Eur. J. Cancer 45, 228-247 (2009) 
17. E. Modigliani, R. Cohen, J.M. Campos, B. Conte-Devolx, B. Maes, A. Boneu, M. Schlumberger, J.C. Bigorgne, P. Dumontier, L. Leclerc, B. Corcuff, I. Guilhem, Prognostic factors for survival and for biochemical cure in medullary thyroid carcinoma: results in 899 patients. The GETC Study Group. Groupe d'etude des tumeurs a calcitonine. Clin. Endocrinol. 48, 265-273 (1998)

18. R. Elisei, M.J. Schlumberger, S.P. Muller, P. Schoffski, M.S. Brose, M.H. Shah, L. Licitra, B. Jarzab, V. Medvedev, M.C. Kreissl, B. Niederle, E.E. Cohen, L.J. Wirth, H. Ali, C. Hessel, Y. Yaron, D. Ball, B. Nelkin, S.I. Sherman, Cabozantinib in progressive medullary thyroid cancer. J. Clin. Oncol. 31, 3639-3646 (2013)

19. S.A. Wells Jr., B.G. Robinson, R.F. Gagel, H. Dralle, J.A. Fagin, M. Santoro, E. Baudin, R. Elisei, B. Jarzab, J.R. Vasselli, J. Read, P. Langmuir, A.J. Ryan, M.J. Schlumberger, Vandetanib in patients with locally advanced or metastatic medullary thyroid cancer: a randomized, double-blind phase III trial. J. Clin. Oncol. 30, 134-141 (2012)

20. M.E. Caplin, M. Pavel, J.B. Ćwikła, A.T. Phan, M. Raderer, E. Sedláčková, G. Cadiot, E.M. Wolin, J. Capdevila, L. Wall, G. Rindi, A. Langley, S. Martinez, J. Blumberg, P. Ruszniewski; CLARINET Investigators., Lanreotide in metastatic enteropancreatic neuroendocrine tumors. N. Engl. J. Med. 371, 1556-1557 (2014)

21. A. Rinke, H.H. Muller, C. Schade-Brittinger, K.J. Klose, P. Barth, M. Wied, C. Mayer, B. Aminossadati, U.F. Pape, M. Blaker, J. Harder, C. Arnold, T. Gress, R. Arnold; PROMID Study Group.,
Placebo-controlled, double-blind, prospective, randomized study on the effect of octreotide LAR in the control of tumor growth in patients with metastatic neuroendocrine midgut tumors: a report from the PROMID Study Group. J. Clin. Oncol. 27, 4656-4663 (2009)

22. S. Grozinsky-Glasberg, H. Rubinfeld, Y. Nordenberg, A. Gorshtein, M. Praiss, E. Kendler, R. Feinmesser, A.B. Grossman, I. Shimon, The rapamycin-derivative RAD001 (everolimus) inhibits cell viability and interacts with the Akt-mTOR-p70S6K pathway in human medullary thyroid carcinoma cells. Mol. Cell. Endocrinol. 315, 87-94 (2010)

23. S. Grozinsky-Glasberg, G. Franchi, M. Teng, C.A. Leontiou, A. Ribeiro de Oliveira Jr., P. Dalino, N. Salahuddin, M. Korbonits, A.B. Grossman, Octreotide and the mTOR inhibitor RAD001 (everolimus) block proliferation and interact with the Akt-mTORp70S6K pathway in a neuro-endocrine tumour cell Line. Neuroendocrinology 87, 168-181 (2008)

24. K.C. Bible, V.J. Suman, J.R. Molina, R.C. Smallridge, W.J. Maples, M.E. Menefee, J. Rubin, N. Karlin, K. Sideras, J.C. Morris 3rd, B. McIver, I. Hay, V. Fatourechi, J.K. Burton, K.P. Webster, C. Bieber, A.M. Traynor, P.J. Flynn, B. Cher Goh, C.R. Isham, P. Harris, C. Erlichman; Endocrine Malignancies Disease Oriented Group, Mayo Clinic Cancer Center, and the Mayo Phase 2 Consortium., A multicenter phase 2 trial of pazopanib in metastatic and progressive medullary thyroid carcinoma: MC057H. J. Clin. Endocrinol. Metab. 99, 1687-1693 (2014) 